\title{
Protective Effect of Metformin Against Thyroid Cancer Development: A Population-Based Study in Korea
}

\author{
Yoon Young Cho,, ${ }^{1,2}$ Min Jin Kang, Soo Kyoung Kim,,2 Jung Hwa Jung,,2 Jong Ryeal Hahm,,2 \\ Tae Hyuk Kim, Joo Young Nam, Byung-Wan Lee, ${ }^{6}$ Yong-ho Lee, Jae Hoon Chung, \\ Sun Ok Song, ${ }^{5, *}$ and Sun Wook Kim, ${ }^{4, *}$
}

\begin{abstract}
Background: Metformin, the most widely used drug for type 2 diabetes, has recently attracted attention with regard to its antitumor activity. However, clinical studies have yielded conflicting results regarding the association between metformin and thyroid cancer development, despite its antitumor effect in preclinical studies. Methods: This is a retrospective cohort study using the Korean National Health Insurance claim database. Matched populations of 128,453 metformin users and 128,453 non-users were analyzed for thyroid cancer incidence. Metformin users were categorized into lowest, middle, and highest tertiles according to cumulative dose or duration of metformin therapy.

Results: Thyroid cancer developed in $340(0.26 \%)$ metformin users and $487(0.38 \%)$ non-users during a mean follow-up of 7.2 years (hazard ratio $=0.69$ [confidence interval $0.60-0.79$ ]; $p<0.001$ ). The incidence of thyroid cancer per $10^{5}$ person-years was 51.6 in metformin non-users. For metformin users, the incidence was 84.5 for $<529,000 \mathrm{mg}, 20.6$ for $529,000-1,007,799 \mathrm{mg}$, and 6.3 for $>1,007,799 \mathrm{mg} ; 86.3$ for $<1085$ days, 20.3 for $1085-$ 2094 days, and 4.7 for $>2094$ days for duration of therapy. The hazard ratio for thyroid cancer decreased significantly in metformin users as a function of dose and duration of metformin therapy.

Conclusions: Metformin appears to be associated with a preventive effect on thyroid cancer development in a nationwide population-based study, but is not effective in the early phase of treatment. Considering the increasing prevalence of obesity and the role of insulin resistance in the development of cancer, metformin might be the preferred treatment for its dual anti-diabetic and antitumor effects.
\end{abstract}

Keywords: metformin, thyroid cancer, protective effect, population-based study

\section{Introduction}

D IABETES IS THE MOST prevalent disease in endocrinology and is associated with an increased risk of cancer development. The incidence of several types of cancers, including colon (1), breast (2), and pancreas (3) cancer, is known to be elevated in patients with type 2 diabetes. A recent study reported a greater risk of all-cause cancers in middle-aged diabetic patients (4). The incidence of thyroid cancer has rapidly increased worldwide in recent decades $(5,6)$. Although the introduction of imaging studies such as ultrasonography have contributed to the detection of small thyroid cancers (5), the increase in thyroid cancers is not fully explained by increased diagnosis and screening alone (6). Other factors such as insulin resistance have been suggested to be factors contributing to cancer development $(7,8)$ as the prevalence of obesity and diabetes increase. The diagnosis of a malignancy adds an additional burden for diabetic individuals and their societies.

Metformin is the most widely used drug for the treatment of type 2 diabetes, with approximately 120 million prescriptions per year globally (9). Aside from its anti-diabetic

${ }^{1}$ Division of Endocrinology and Metabolism, Department of Medicine; ${ }^{2}$ Institute of Health Sciences; Gyeongsang National University School of Medicine, Jinju, Korea.

${ }^{3}$ Department of Policy Research Affairs, National Health Insurance Service Ilsan Hospital, Goyang, Korea.

${ }^{4}$ Division of Endocrinology and Metabolism, Department of Medicine, Thyroid Center, Samsung Medical Center, Sungkyunkwan University School of Medicine, Seoul, Korea.

${ }_{5}^{5}$ Division of Endocrinology and Metabolism, Department of Internal Medicine, National Health Insurance Service Ilsan Hospital, Goyang, Korea.

${ }^{6}$ Division of Endocrinology and Metabolism, Department of Internal Medicine, Severance Hospital, Yonsei University College of Medicine, Seoul, Korea.

*Sun Wook Kim and Sun Ok Song are joint senior authors. 
effect, experimental studies have revealed antitumor effects of metformin. Metformin reduces circulating insulin by enhancing insulin sensitivity and decreasing hepatic gluconeogenesis. In addition, metformin inhibits the mammalian target of rapamycin (mTOR) pathway via activation of AMPactivated protein kinase (AMPK), which leads to the inhibition of protein synthesis and cell proliferation (10). Previous studies observed that metformin inhibits thyroid cancer cell growth and induces cell-cycle arrest and apoptosis through AMPK activation and mTOR inhibition in vivo and in vitro $(11,12)$.

However, epidemiologic studies examining the antitumor effect of metformin on thyroid cancer failed to give consistent results. Tseng et al. reported a protective effect of metformin on thyroid cancer risk in a Taiwanese population with type 2 diabetes compared to metformin never-users (hazard ratio $[\mathrm{HR}]=0.683$ [confidence interval (CI) 0.598-0.780]) (13). To date, this study is the only epidemiologic study reporting the protective effect of metformin on thyroid cancer development, although their results were weakened by insufficient definition of metformin user and duration of drug exposure. Two subsequent studies conducted in Western populations observed no relationship between metformin exposure and thyroid cancer development $(14,15)$. The small proportion of diabetic subjects in their studies- $5.7 \%$ (14) and $15.8 \%$ (15), respectivelycould be the reason for the results.

There is less evidence for the beneficial effect of metformin on thyroid cancer development than for other types of cancer. Therefore, this study investigated the association between metformin exposure and thyroid cancer incidence in newly diagnosed type 2 diabetic individuals using the National Health Insurance (NHI) claim database in Korea, which covers the entire population.

\section{Methods}

\section{Data source}

Data from the NHI claim database of Korea were used. The $\mathrm{NHI}$ is the only public medical insurance system in Korea and represents the entire Korean population because of the compulsory social insurance system that is required by law. All clinics and hospitals in Korea submit data on inpatients and outpatients, including information on diagnosis and medical costs, to the NHI to claim payments for patient care (16).

\section{Study population}

This study enrolled newly diagnosed diabetic subjects aged $\geq 30$ years who had been prescribed anti-diabetic drug(s) at least twice between January 2005 and December 2009. To enroll subjects with new-onset diabetes only, individuals with previous claims for any anti-diabetic drugs or a coding of diabetes in a three-year washout period before January 2005 were excluded. Subjects with claims for insulin prescriptions more than twice in the study period were also excluded to rule out the possible effect of insulin on the development and growth of thyroid cancer, as were individuals who had been followed up for $<180$ days. The protocol was reviewed and approved by the Institutional Review Board of National Health Insurance Service Ilsan Hospital (NHIMC 2016-03-004).

\section{Definition of metformin exposure}

Metformin users were defined as subjects who had been prescribed with metformin for at least 180 days during 365 days. Metformin non-users were defined as subjects who had never been prescribed metformin or who did not satisfy the definition of metformin user (metformin use for $<180$ days) during the whole study period (from January 2005 to December 2014). Among 570,759 metformin users and 145,481 metformin non-users, the users and non-users were matched by five covariates: age, sex, income, living area, and anti-diabetic drugs other than metformin. This resulted in 128,453 metformin users and 128,453 metformin non-users for analysis in this study. To assess the effect of metformin on thyroid cancer incidence according to cumulative dose and duration, the sum of prescribed dose ( $\mathrm{mg}$ per day $\times$ days) and the duration (days) of metformin use in each person were calculated, and subjects were divided into three groups (tertiles) for prescribed dose or duration.

\section{Detection of thyroid cancer}

Thyroid cancer is classified with code C73.9 according to the International Classification of Diseases, 10th edition (ICD-10) (17). Diagnosis of thyroid cancer was defined by a claim for any type of thyroidectomy with a coding of thyroid cancer between January 2005 and December 2014. To assess the incidence of newly developed thyroid cancer, individuals who had claimed for thyroid cancer before study enrollment were excluded. Subjects with claims for thyroid cancer before the diagnosis of diabetes were also excluded. Follow-up duration was calculated from the date of study enrollment to diagnosis of thyroid cancer or death or the end date of this study, which was December 2014.

\section{Covariates}

Age groups were categorized using five-year intervals starting at 30 years of age. As newly diagnosed diabetic patients were selected using a three-year washout period, the year of enrollment was regarded as the year of diagnosis with diabetes or the beginning of anti-diabetic medication. Income groups were divided into 20 classes and reclassified into four groups. Living area was classified into three groups: Seoul (capital city), metropolitan city (Busan, Daegu, Incheon, Gwangju, Daejeon, and Ulsan), and rural area. Anti-diabetic drugs other than metformin included sulfonylureas, thiazolidinediones, dipeptidyl peptidase-4 inhibitors, and alpha glucosidase inhibitors. Information for these drugs was assessed by claim records.

\section{Statistical analysis}

Subjects were classified into two groups: metformin users and metformin non-users. The incidence of thyroid cancer is presented as number of events per 100,000 person-years (PY). The HR and CI between two groups of interest were calculated using Cox proportional hazards regression, with adjustment for age, sex, year of study enrollment, income, living area, and anti-diabetic drugs other than metformin. A $p$-value of $<0.05$ was considered to indicate a statistically significant result. All analyses were conducted using statistical software SAS v9.4 (SAS Institute, Cary, NC). 


\section{Results}

\section{Demographics}

This study consisted of 128,453 metformin non-users and 128,453 metformin users. Their demographics, including age, sex, year of study enrollment, income, living area, and anti-diabetic drugs other than metformin, are presented in Table 1. Metformin users tended to be older $\left(M_{\text {age }}=62\right.$ years vs. 61 years), female ( $50.4 \%$ vs. $48.8 \%)$, earn more income, and live in a capital city compared to metformin non-users. In metformin users, the $M \pm S D$ daily prescribed dose of metformin was $531 \pm 161 \mathrm{mg}, M \pm S D$ cumulative duration was $1633 \pm 915$ days, and $M \pm S D$ cumulative dose was $868,169 \pm 563,221 \mathrm{mg}$. The duration between starting metformin and diagnosis of thyroid cancer was longer in metformin users than that of metformin non-users (37.2 vs. 31.5 months).

\section{Thyroid cancer incidence in diabetic subjects according to metformin use}

During a 7.2-year mean follow-up duration in each group, $487(0.38 \%)$ thyroid cancers were detected in metformin nonusers compared to $340(0.26 \%)$ in metformin users. The incidence rate of thyroid cancer per 100,000 PY was 51.6 in the metformin non-users (reference) and 36.1 in the metformin users. Multiple-adjusted HR indicated that metformin users had a lower risk for thyroid cancer incidence than metformin non-users (HR =0.69 [CI 0.60-0.79]; $p<0.001$; Table 2).

When calculating HR according to cumulative dose and duration of metformin, a dose- or duration-dependent protective effect on thyroid cancer development was observed. Risk for thyroid cancer was reduced in metformin users with middle $(529,000-1,007,799 \mathrm{mg})$ or highest tertiles $(>1,007,799 \mathrm{mg})$ of cumulative dose compared to metformin non-users $(\mathrm{HR}=0.37$ [CI 0.28-0.48]; $p<0.001$; and $\mathrm{HR}=0.11$ [CI 0.07-0.17]; $p<0.001)$. Users who had been prescribed metformin for a longer duration developed thyroid cancer less frequently than metformin non-users (cumulative days of metformin use 1085-2094 days; $\mathrm{HR}=0.36$ [CI 0.27-0.47]; $p<0.001$; and $>2094$ days; $\mathrm{HR}=0.08$ [CI 0.05-0.13]; $p<0.001)$. However, the lowest tertiles of cumulative metformin dose $(<529,000 \mathrm{mg})$ or duration $(<1085$ days $)$ were at higher risk of thyroid cancer incidence compared to metformin non-users $(\mathrm{HR}=1.79$ [CI 1.53-2.08]; $p<0.001$; and $\mathrm{HR}=1.86$ [CI 1.60-2.16]; $p<0.001)$. The chi-square test for trend showed a significant association between dose or duration of metformin use and the development of thyroid cancer (both $p<0.001$ ). Thyroid cancer risks were reduced significantly in both sexes (male: $\mathrm{HR}=0.68$ [CI $0.51-0.92] ; p=0.01$; and female: $\mathrm{HR}=0.65$ [CI $0.56-0.76] ; p<0.001)$ and in middle-aged subjects (aged 50-64 years; Supplementary Tables S1 and S2).

\section{Discussion}

This study examined the protective effect of metformin on thyroid cancer development based on a nationwide population-based database. A significant association was found between the use of metformin and thyroid cancer, especially in diabetic individuals taking metformin with a higher cumulative dose $(\geq 529,000 \mathrm{mg})$ or for a longer duration ( $\geq 1085$ days). In contrast, no protective effect was observed for a lower cumulative dose or during the early period of metformin use.
A considerable number of studies demonstrated the anticarcinogenic effect of metformin in thyroid cancer development using preclinical data. Chen et al. reported an anti-mitogenic effect of metformin on thyroid cancer cells and thyroid cancer stem cells through inhibition of cell-cycle progression and induction of apoptosis (11). Cho et al. suggested the therapeutic potential of a physiologic dose of metformin in vivo and in vitro (12). The viability of thyroid cancer cells was inhibited and cell apoptosis was increased for various doses of metformin (0.5-20 mM), and the p-AMPK/AMPK ratio was activated and mTOR expression was suppressed in metformin-treated mice compared to control mice. The antitumor effect of metformin occurs by direct and indirect mechanisms (18). Signaling of mTOR induces protein synthesis and tumorigenesis, and metformin inhibits mTOR activity directly or via AMPK activation. In addition, metformin increases insulin sensitivity and suppresses hepatic gluconeogenesis, which results in a decreased level of circulating insulin. As a high concentration of serum insulin stimulates mTOR signaling, metformin is expected to inhibit mTOR expression in an indirect manner.

In contrast to preclinical research, clinical studies regarding the antitumor effect of metformin on thyroid cancer have presented conflicting results. Tseng et al. reported a $32 \%$ risk reduction of thyroid cancer in patients with metformin exposure in a Taiwanese population (13). Their results are comparable to the present data (a $31 \%$ risk reduction). However, they observed a diminished risk of thyroid cancer in the early period of metformin exposure $(<9$ months or $<263,000 \mathrm{mg}$ of metformin use). Although six-month treatment with metformin induced a remarkable volume reduction of benign thyroid nodules (median reduction of $108.5 \mathrm{~mm}^{3}$, $-30 \%, p<0.008)$ in a prospective study $(n=14)(19)$, the effect of short-term use of metformin on thyroid nodules, including carcinomas, needs to be investigated considering that the natural growth of thyroid nodules is $<1 \mathrm{~mm}$ growth in the largest diameter per year (20). The previous study by Tseng et al. has been criticized for the study design, as they defined metformin exposure based on prescription history prior to study entry and did not assess changes in metformin exposure during the study period. In the present study, the authors tried to examine the exact dose and duration of metformin exposure in each subject using a reliable claim database. Two consecutive studies, a case control study (14) and a prospective cohort study (15), reported no association between metformin and thyroid cancer risk $(\mathrm{HR}=1.48[\mathrm{CI}$ 0.86-2.54] and HR = 1.09 [CI 0.79-1.52], respectively). These well-designed studies considered covariates for thyroid cancer such as alcohol consumption, smoking, and obesity. However, the majority of their study populations comprised non-diabetic individuals. Thus, the small number of diabetic patients with metformin exposure brings the negative results of their studies into question.

The present data demonstrate a protective effect of metformin on thyroid cancer development in a dose- or durationdependent manner. For example, three-year use of metformin with $500 \mathrm{mg}$ per day is expected to have a preventive effect for thyroid cancer. Interestingly, metformin users had a longer duration between starting metformin and thyroid cancer diagnosis than metformin non-users (mean duration of 37.2 months vs. 31.5 months), possibly supporting a preventive effect of metformin on thyroid cancer development. 
Table 1. Demographics of Subjects According to Metformin Use

\begin{tabular}{|c|c|c|c|c|}
\hline \multirow[b]{2}{*}{ Variables } & \multicolumn{2}{|c|}{$\begin{array}{c}\text { Metformin } \\
\text { non-user }(\mathrm{N}=128,453)\end{array}$} & \multicolumn{2}{|c|}{$\begin{array}{c}\text { Metformin } \\
\text { user }(\mathrm{N}=128,453)\end{array}$} \\
\hline & $\mathrm{n}$ & $\%$ & $\mathrm{n}$ & $\%$ \\
\hline Age (years), $M \pm S D^{*}$ & \multicolumn{2}{|c|}{$61 \pm 15$} & \multicolumn{2}{|c|}{$62 \pm 14$} \\
\hline $30-34$ & 3523 & 2.8 & 1535 & 1.2 \\
\hline $35-39$ & 4973 & 3.9 & 3661 & 2.8 \\
\hline $40-44$ & 7784 & 6.1 & 6661 & 5.2 \\
\hline $45-49$ & 12,252 & 9.5 & 11,513 & 9.0 \\
\hline $50-54$ & 14,092 & 11.0 & 14,580 & 11.4 \\
\hline $55-59$ & 15,093 & 11.7 & 16,305 & 12.7 \\
\hline $60-64$ & 16,219 & 12.6 & 17,924 & 14.0 \\
\hline $65-69$ & 18,022 & 14.0 & 19,811 & 15.4 \\
\hline $70-74$ & 15,731 & 12.2 & 16,632 & 12.9 \\
\hline $75-79$ & 11,527 & 9.0 & 11,419 & 8.9 \\
\hline$\geq 80$ & 9237 & 7.2 & 8412 & 6.5 \\
\hline Sex, male* & 65,746 & 51.2 & 63,733 & 49.6 \\
\hline \multicolumn{5}{|l|}{ Year of study enrollment* } \\
\hline 2005 & 30,471 & 23.7 & 28,251 & 22.0 \\
\hline 2006 & 26,389 & 20.6 & 25,480 & 19.8 \\
\hline 2007 & 25,053 & 19.5 & 25,498 & 19.9 \\
\hline 2008 & 24,570 & 19.1 & 25,874 & 20.1 \\
\hline 2009 & 21,970 & 17.1 & 23,350 & 18.2 \\
\hline Thyroid cancer cases* & 487 & 0.38 & 340 & 0.26 \\
\hline \multicolumn{5}{|l|}{ Year of diagnosis of thyroid cancer* } \\
\hline 2005 & 2 & 0.4 & 0 & 0 \\
\hline 2006 & 25 & 5.1 & 15 & 4.4 \\
\hline 2007 & 85 & 17.5 & 31 & 9.1 \\
\hline 2008 & 149 & 30.6 & 70 & 20.6 \\
\hline 2009 & 84 & 17.2 & 76 & 22.4 \\
\hline 2010 & 42 & 8.6 & 37 & 10.9 \\
\hline 2011 & 36 & 7.4 & 35 & 10.3 \\
\hline 2012 & 26 & 5.3 & 35 & 10.3 \\
\hline 2013 & 26 & 5.3 & 21 & 6.2 \\
\hline 2014 & 12 & 2.5 & 20 & 5.9 \\
\hline Follow-up duration (years), $M \pm S D$ & 7.2 & 1.8 & 7.2 & 1.6 \\
\hline $\begin{array}{l}\text { Duration between starting metformin and } \\
\quad \text { diagnosis of thyroid cancer (months), } M \pm \mathrm{SD}^{*}\end{array}$ & 31.5 & 21.1 & 37.2 & 23.2 \\
\hline \multicolumn{5}{|l|}{ Income* } \\
\hline $1-5$ (lowest) & 39,472 & 30.7 & 37,885 & 29.5 \\
\hline $6-10$ & 23,625 & 18.4 & 23,255 & 18.1 \\
\hline $11-15$ & 29,462 & 22.9 & 29,750 & 23.2 \\
\hline 16-20 (highest) & 35,894 & 28.0 & 37,563 & 29.2 \\
\hline \multicolumn{5}{|l|}{ Living area* } \\
\hline Seoul (capital city) & 27,606 & 21.5 & 28,734 & 22.4 \\
\hline Metropolitan city & 32,127 & 25.0 & 32,137 & 25.0 \\
\hline Rural area & 68,720 & 53.5 & 67,582 & 52.6 \\
\hline \multicolumn{5}{|l|}{ Anti-diabetic drugs } \\
\hline \multicolumn{5}{|l|}{ Sulfonylurea* } \\
\hline Yes & 61,172 & 47.6 & 62,679 & 48.8 \\
\hline No & 67,281 & 52.4 & 65,774 & 51.2 \\
\hline \multicolumn{5}{|l|}{ Thiazolidinedione* } \\
\hline Yes & 5228 & 4.1 & 5426 & 4.2 \\
\hline No & 123,225 & 95.9 & 123,027 & 95.8 \\
\hline \multicolumn{5}{|l|}{ DPP-4 inhibitor* } \\
\hline Yes & 2375 & 1.8 & 2460 & 1.9 \\
\hline No & 126,078 & 98.2 & 125,993 & 98.1 \\
\hline \multicolumn{5}{|l|}{ Alpha glucosidase inhibitor* } \\
\hline Yes & 11,403 & 8.9 & 10,404 & 8.1 \\
\hline No & 117,050 & 91.1 & 118,049 & 91.9 \\
\hline
\end{tabular}

Variables are presented as number $(n)$ and percentage (\%), except for age, follow-up duration, and the duration between starting metformin and diagnosis of thyroid cancer. Metformin non-users were defined as subjects who had never been prescribed with metformin or did not satisfy the definition of metformin user during the study period. Metformin users were defined as subjects who had been prescribed with metformin for at least 180 days during 365 days. Follow-up duration was similar in metformin users and non-users $(p=0.49)$. $* p<0.001$.

$S D$, standard deviation; DPP-4 inhibitor, dipeptidyl peptidase-4 inhibitor. 
Table 2. Hazard Ratio for Thyroid Cancer Incidence in Diabetic Subjects by Metformin Use

\begin{tabular}{|c|c|c|c|c|c|c|}
\hline Metformin use & $\begin{array}{l}\text { Diabetic } \\
\text { subjects }\end{array}$ & $\begin{array}{c}\text { Thyroid cancer } \\
\text { case }\end{array}$ & $\begin{array}{l}\text { Person- } \\
\text { year }\end{array}$ & $\begin{array}{c}\text { Incidence } \\
\text { rate of thyroid } \\
\text { cancer }(\%)\end{array}$ & $\begin{array}{l}\text { Incidence of } \\
\text { thyroid cancer } \\
\text { (per 100,000 } \\
\text { person-years) }\end{array}$ & $\begin{array}{l}\text { Hazard ratio } \\
\text { of thyroid } \\
\text { cancer }[C I]\end{array}$ \\
\hline Non-user & 128,453 & 487 & 943,036 & 0.38 & 51.6 & Reference \\
\hline User & 128,453 & 340 & 941,334 & 0.26 & 36.1 & $0.69[0.60-0.79]^{*}$ \\
\hline \multicolumn{7}{|c|}{ Cumulative dose (mg) } \\
\hline$<529,000$ & 41,921 & 256 & 302,812 & 0.61 & 84.5 & $1.79[1.53-2.08]^{*}$ \\
\hline $\begin{array}{l}529,000- \\
1,007,799\end{array}$ & 43,566 & 63 & 305,157 & 0.14 & 20.6 & $0.37[0.28-0.48]^{*}$ \\
\hline$>1,007,799$ & 42,966 & 21 & 333,365 & 0.05 & 6.3 & $0.11[0.07-0.17]^{*}$ \\
\hline \multicolumn{7}{|c|}{ Cumulative duration of therapy (days) } \\
\hline$<1085$ & 42,218 & 264 & 305,889 & 0.63 & 86.3 & $1.86[1.60-2.16]^{*}$ \\
\hline $1085-2094$ & 42,777 & 60 & 295,829 & 0.14 & 20.3 & $0.36[0.27-0.47]^{*}$ \\
\hline$>2094$ & 43,458 & 16 & 339,616 & 0.04 & 4.7 & $0.08[0.05-0.13]^{*}$ \\
\hline
\end{tabular}

Metformin non-users were defined as subjects who had never been prescribed with metformin or did not satisfy the definition of metformin user during the study period. Metformin users were defined as subjects who had been prescribed with metformin for at least 180 days during 365 days. Hazard ratio was adjusted by age, sex, year of study enrollment, income, living area, and anti-diabetic drugs other than metformin.

$* p<0.001$, compared with metformin non-user (reference).

CI, confidence interval.

Lin et al. also reported a dose-dependent effect of metformin on cancer risk and cancer onset time using a longitudinal health insurance database. Diminished cancer risk was significant with a $>360$ defined daily dose (equal to $720,000 \mathrm{mg}$ ) of metformin ( $\mathrm{HR}=0.40$ [CI $0.24-0.66] ; p<0.001)$ compared to diabetic patients who did not receive anti-diabetic medication (21), and their results were comparable with the data in the present study, although they did not analyze the cancer risk by cancer subtype.

The majority of thyroid cancers are detected as small masses and have a good prognosis, which has led to changes in the concept of treatment toward adopting less aggressive surgery, including lobectomy only (22). Moreover, active surveillance is suggested as one of the treatment strategies for early-stage thyroid cancers in selected patients instead of surgery (22). Inhibition of development or progression of thyroid cancer can keep tumors in an indolent state, and in this regard, metformin might be a promising candidate because of its antitumor effect. Because the effect of metformin is not as potent as that of therapeutic agents used for chemotherapy, demonstrating a preventive effect against carcinogenesis is challenging, especially in studies with insufficient sample sizes $(14,15)$, which has resulted in controversies. Whether metformin delays sprouting or progression of thyroid cancer might be an interesting topic for further studies.

These promising results cannot be applied to all diabetic subjects because of the varied disease status of diabetes and the effect of other medications, including insulin. Patients with advanced diabetes and comorbidities usually take multiple medications. In these patients, disease status, as well as medications, may affect sensitivity to insulin action or cancer development. Some authors have suggested a relationship between elevated risks of cancers and insulin or sulfonylurea therapy, probably due to their effects of increasing insulin levels $(23,24)$. However, Lin et al. reported a reduced cancer risk, even in patients taking metformin combined with other anti-diabetic medication (21). In another study by Tseng et al., a reduced risk for thyroid cancer was reported in subjects with early diabetes (duration of diabetes $<5$ years), whereas the effect was neutralized in individuals with a longer diabetes duration (23). Thus, the study subjects were limited to people who had newly diagnosed diabetes or started taking anti-diabetic medication except for insulin to minimize the heterogeneity of the study population.

An increased risk of thyroid cancer was observed in the early period of metformin use. As metformin reduces insulin resistance and has a neutral effect on weight gain, metformin is preferred for obese diabetic patients. The prevalence of obesity in the Korean population has increased rapidly, especially in adult men (body mass index $[\mathrm{BMI}] \geq 25 \mathrm{~kg} / \mathrm{m}^{2}$, from $32.4 \%$ in 2001 to $36.7 \%$ in 2007) (25). Obesity has been suggested as one of the risk factors for thyroid cancer (26). In fact, the clinical characteristics of metformin users can be linked with risk factors of thyroid cancer. In a mouse model, a high-fat diet aggravates thyroid cancer progression via activation of the leptin-JAK2-STAT3 signaling pathway (27), whereas metformin blocks progression of obesity-activated thyroid cancer (28). It was cautiously assumed that the demographics of metformin users, including obesity, also increase the risk of thyroid cancer, and that the decreased insulin resistance due to metformin may have a protective effect on cancer development, although data for BMI or obesity were not available. Metformin users had a tendency to earn a higher income and live in the capital city than metformin non-users, and these socioeconomic factors might promote thyroid cancer detection through thyroid screening. Due to the limitation of the retrospective design and the prevalence of thyroid screening in Korea during the study period, an uncorrectable bias caused by thyroid screening may affect the thyroid cancer incidence, especially in the early phase of metformin use. However, an attempt was made to adjust covariates in multiple regression analysis, and it was found that the protective effect of metformin remained significant.

In subgroup analysis, both females and males, especially middle-aged diabetic subjects, gained a protective effect from metformin on thyroid cancer development. Previous 
studies, including epidemiologic data (13-15), did not suggest specific groups that may benefit from metformin in terms of thyroid cancer development. The age-specific effect of metformin on cancer development has not been reported in the literature, and both diabetes and thyroid cancer are prevalent in middle-aged subjects (aged 50-64 years). Thus, the preventive effect of metformin seems to reach a statistically significant value in middle-aged subjects compared to other age groups.

This study had several limitations. First, due to its retrospective design and the limitation of the NHI claim data set, it was not possible to control for biases or obtain additional information related to thyroid cancer incidence such as screening of thyroid disease, personal or family history of thyroid disease, drinking, smoking habits, and obesity. To set a comparable control group, metformin non-users rather than healthy subjects were defined as controls with consideration of shared common characteristics among the diabetic population. In addition, metformin users and non-users were matched at baseline, and a multiple regression test was used adjusting for available parameters. Second, this study has a relatively short follow-up duration considering the indolent progression of thyroid cancer. Clinical studies for the protective effect of metformin on thyroid cancer are scarce. In an earlier study, Gwiezdzinska et al. reported a smaller tumor size and a higher remission rate of thyroid cancer in diabetic patients taking metformin, with a 4.4-year of mean treatment than metformin non-users (29). Insulin users were excluded, and the present data have a short follow-up duration (7.2 years) compared to two prospective studies of 11 (14) and 15 years (15). However, the present data have a longer PY duration $(1,884,370 \mathrm{PY})$. Third, the effect of metformin on aggressiveness of thyroid cancer could not be assessed because of the lack of information on cancer histology, stage, or disease-specific death, although this issue is beyond the scope of this study. Two studies suggested that metformin use was related to a better prognosis of thyroid cancer, a higher remission rate (29), and a lower recurrence rate of cervical lymph node metastasis (30) in thyroid cancer patients, but this needs to be validated in future studies. Finally, information on drug adherence was not available, although suboptimal adherence for anti-diabetic drugs has been reported in patients with type 2 diabetes (31).

In conclusion, this study reports a significant association between metformin use and a decreased risk of thyroid cancer development using a nationwide population-based database. As the first choice of anti-diabetic drug in the majority of new patients with type 2 diabetes, metformin is expected to have a preventive effect on the development of thyroid cancer in addition to its glucose-lowering effect.

\section{Acknowledgments}

This study was supported by a grant from the National Health Insurance Service Policy Research (2016-20-011). The funding source was not involved in oversight or design of the study, in the analysis or interpretation of the data, or in the decision to submit the manuscript for publication.

\section{Author Disclosure Statement}

The authors have no competing interests related to this study to declare.

\section{References}

1. Guraya SY 2015 Association of type 2 diabetes mellitus and the risk of colorectal cancer: a meta-analysis and systematic review. World J Gastroenterol 21:6026-6031.

2. Boyle P, Boniol M, Koechlin A, Robertson C, Valentini F, Coppens K, Fairley LL, Boniol M, Zheng T, Zhang Y, Pasterk M, Smans M, Curado MP, Mullie P, Gandini S, Bota M, Bolli GB, Rosenstock J, Autier P 2012 Diabetes and breast cancer risk: a meta-analysis. $\mathrm{Br} \mathrm{J}$ Cancer 107: 1608-1617.

3. Ben Q, Xu M, Ning X, Liu J, Hong S, Huang W, Zhang H, Li Z 2011 Diabetes mellitus and risk of pancreatic cancer: a meta-analysis of cohort studies. Eur J Cancer 47:19281937.

4. Yang WS, Chen PC, Lin HJ, Su TC, Hsu HC, Chen MF, Lee YT, Chien KL 2017 Association between type 2 diabetes and cancer incidence in Taiwan: data from a prospective community-based cohort study. Acta Diabetol 54: 455-461.

5. Morris LG, Sikora AG, Tosteson TD, Davies L 2013 The increasing incidence of thyroid cancer: the influence of access to care. Thyroid 23:885-891.

6. Lim H, Devesa SS, Sosa JA, Check D, Kitahara CM 2017 Trends in thyroid cancer incidence and mortality in the United States, 1974-2013. JAMA 317:1338-1348.

7. Kitahara CM, Platz EA, Freeman LE, Hsing AW, Linet MS, Park Y, Schairer C, Schatzkin A, Shikany JM, Berrington de Gonzalez A 2011 Obesity and thyroid cancer risk among U.S. men and women: a pooled analysis of five prospective studies. Cancer Epidemiol Biomarkers Prev 20: 464-472.

8. Yeo Y, Ma SH, Hwang Y, Horn-Ross PL, Hsing A, Lee KE, Park YJ, Park DJ, Yoo KY, Park SK 2014 Diabetes mellitus and risk of thyroid cancer: a meta-analysis. PLoS One 9:e98135.

9. Ben Sahra I, Le Marchand-Brustel Y, Tanti JF, Bost F 2010 Metformin in cancer therapy: a new perspective for an old antidiabetic drug? Mol Cancer Ther 9:1092-1099.

10. Zhou G, Myers R, Li Y, Chen Y, Shen X, Fenyk-Melody J, Wu M, Ventre J, Doebber T, Fujii N, Musi N, Hirshman MF, Goodyear LJ, Moller DE 2001 Role of AMP-activated protein kinase in mechanism of metformin action. J Clin Invest 108:1167-1174.

11. Chen G, Xu S, Renko K, Derwahl M 2012 Metformin inhibits growth of thyroid carcinoma cells, suppresses selfrenewal of derived cancer stem cells, and potentiates the effect of chemotherapeutic agents. J Clin Endocrinol Metab 97:E510-520.

12. Cho SW, Yi KH, Han SK, Sun HJ, Kim YA, Oh BC, Park YJ, Park DJ 2014 Therapeutic potential of metformin in papillary thyroid cancer in vitro and in vivo. Mol Cell Endocrinol 393:24-29.

13. Tseng $\mathrm{CH} 2014$ Metformin reduces thyroid cancer risk in Taiwanese patients with type 2 diabetes. PLoS One 9: e109852.

14. Becker C, Jick SS, Meier CR, Bodmer M 2015 No evidence for a decreased risk of thyroid cancer in association with use of metformin or other antidiabetic drugs: a case-control study. BMC Cancer 15:719.

15. Luo J, Phillips L, Liu S, Wactawski-Wende J, Margolis KL 2016 Diabetes, diabetes treatment, and risk of thyroid cancer. J Clin Endocrinol Metab 101:1243-1248.

16. Song SO, Jung CH, Song YD, Park CY, Kwon HS, Cha BS, Park JY, Lee KU, Ko KS, Lee BW 2014 Background and 
data configuration process of a nationwide populationbased study using the Korean National Health Insurance system. Diabetes Metab J 38:395-403.

17. Fritz A, Percy C, Jack A, Shanmugaratnam K, Sobin L, Parkin DM, S W 2000 International Classification of Diseases for Oncology. World Health Organization, Geneva, Switzerland.

18. Dowling RJ, Goodwin PJ, Stambolic V 2011 Understanding the benefit of metformin use in cancer treatment. BMC Med 9:33.

19. Rezzonico J, Rezzonico M, Pusiol E, Pitoia F, Niepomniszcze H 2011 Metformin treatment for small benign thyroid nodules in patients with insulin resistance. Metab Syndr Relat Disord 9:69-75.

20. Durante C, Costante G, Lucisano G, Bruno R, Meringolo D, Paciaroni A, Puxeddu E, Torlontano M, Tumino S, Attard M, Lamartina L, Nicolucci A, Filetti S 2015 The natural history of benign thyroid nodules. JAMA 313:926935.

21. Lin HC, Kachingwe BH, Lin HL, Cheng HW, Uang YS, Wang LH 2014 Effects of metformin dose on cancer risk reduction in patients with type 2 diabetes mellitus: a 6-year follow-up study. Pharmacotherapy 34:36-45.

22. Haugen BR, Alexander EK, Bible KC, Doherty GM, Mandel SJ, Nikiforov YE, Pacini F, Randolph GW, Sawka AM, Schlumberger M, Schuff KG, Sherman SI, Sosa JA, Steward DL, Tuttle RM, Wartofsky L 20162015 American Thyroid Association Management guidelines for adult patients with thyroid nodules and differentiated thyroid cancer: the American Thyroid Association Guidelines Task Force on Thyroid Nodules and Differentiated Thyroid Cancer. Thyroid 26:1-133.

23. Tseng CH 2012 Thyroid cancer risk is not increased in diabetic patients. PLoS One 7:e53096.

24. Karlstad O, Starup-Linde J, Vestergaard P, Hjellvik V, Bazelier MT, Schmidt MK, Andersen M, Auvinen A, Haukka J, Furu K, de Vries F, De Bruin ML 2013 Use of insulin and insulin analogs and risk of cancer-systematic review and meta-analysis of observational studies. Curr Drug Saf 8:333-348.

25. Khang YH, Yun SC 2010 Trends in general and abdominal obesity among Korean adults: findings from 1998, 2001, 2005, and 2007 Korea National Health and Nutrition Examination Surveys. J Korean Med Sci 25:1582-1588.

26. Oberman B, Khaku A, Camacho F, Goldenberg D 2015 Relationship between obesity, diabetes and the risk of thyroid cancer. Am J Otolaryngol 36:535-541.
27. Kim WG, Park JW, Willingham MC, Cheng SY 2013 Dietinduced obesity increases tumor growth and promotes anaplastic change in thyroid cancer in a mouse model. Endocrinology 154:2936-2947.

28. Park J, Kim WG, Zhao L, Enomoto K, Willingham M, Cheng SY 2016 Metformin blocks progression of obesityactivated thyroid cancer in a mouse model. Oncotarget 7: 34832-34844.

29. Klubo-Gwiezdzinska J, Costello J Jr, Patel A, Bauer A, Jensen K, Mete M, Burman KD, Wartofsky L, Vasko V 2013 Treatment with metformin is associated with higher remission rate in diabetic patients with thyroid cancer. $\mathbf{J}$ Clin Endocrinol Metab 98:3269-3279.

30. Jang EK, Kim WG, Kwon H, Choi YM, Jeon MJ, Kim TY, Shong YK, Kim WB, Kim EY 2015 Metformin is associated with a favorable outcome in diabetic patients with cervical lymph node metastasis of differentiated thyroid cancer. Eur Thyroid J 4:181-188.

31. Iglay K, Cartier SE, Rosen VM, Zarotsky V, Rajpathak SN, Radican L, Tunceli K 2015 Meta-analysis of studies examining medication adherence, persistence, and discontinuation of oral antihyperglycemic agents in type 2 diabetes. Curr Med Res Opin 31:1283-1296.

Address correspondence to: Sun Wook Kim, MD, PhD Division of Endocrinology and Metabolism Department of Medicine Thyroid Center, Samsung Medical Center Sungkyunkwan University School of Medicine 81 Irwon-ro, Gangnam-gu Seoul 06351 Korea

E-mail: swkimmd@skku.edu

Sun Ok Song, MD, PhD Division of Endocrinology and Metabolism Department of Internal Medicine National Health Insurance Service Ilsan Hospital 100 Ilsan-ro, Ilsandong-gu Goyang 10444 Korea

E-mail: songsun7777@gmail.com 